\title{
Impact of different recommendations on adequacy rate for sleep duration in children
}

\author{
Oliviero Bruni ${ }^{1}$ and Paolo Brambilla ${ }^{2,3^{*}}$
}

\begin{abstract}
A huge amount of literature in the last decades showed that sleep is essential for children's health and well-being and that short sleep duration is associated with several negative health outcomes. Many developmental phases in infancy and childhood are in strict relationship with an healthy sleep.

In the last years some specific recommendations made for how much sleep children need have been published. The empirical evidences for contemporary sleep recommendations has changed and the new recommendations are clearly different from the previous ones and reflect clearly the changes in the sleep need of the children and adolescents in the last decades although seem still to be largely unfitting for preadolescence and adolescence. If sleep is to be treated as a therapeutic intervention, then consensus guidelines, statements, and evidence-based best-practice documents are needed to underpin sleep recommendations for children.

Sleep recommendations for children play an important role for public policies and interventions, and to advertise parents and children of the negative consequences of sleep deprivation/reduction.
\end{abstract}

Keywords: Sleep duration, Sleep recommendations, Infants, Children, Prevention

\section{Background}

Sleep is fundamental for children's health and well-being and several studies demonstrated that short sleep duration is associated with several negative physical, emotional, behavioral and cognitive outcomes and with an increased risk of obesity and of psychiatric problems [1-4].

Further, sleep in infancy and early childhood is associated with many important developmental factors, including language development, memory, executive functioning, attention and behavior $[5,6]$.

A recent meta-analysis by Matricciani et al. [7] on 690,747 children reported a decreased sleep duration of 0.75 min per year over the past century, with the rate of change being greatest on school days, for older children, and for boys. This observation is worrying for several reasons: inadequate quality or quantity of sleep in children can have negative impacts on daytime functioning

\footnotetext{
* Correspondence: paolo.brambilla3@gmail.com

${ }^{2}$ Family Pediatrician, Azienda Tutela della Salute (ATS), Città Metropolitana di Milano, Italy

${ }^{3}$ Via Parada 32, 20854 Vedano al Lambro (MB), Italy

Full list of author information is available at the end of the article
}

and there is a clear link between sleep problems at early ages and later behavioral, emotional problems and some aspects of poorer neuropsychological functioning in adolescence $[8,9]$.

\section{Recommendations for sleep}

The results of sleep extension/restriction studies provide evidence for the beneficial or detrimental effect of sleep manipulation but do not give indications on specific hour-based sleep recommendations. A recent study raised the question on whether or not there are sufficient data to support the specific recommendations made for how much sleep children need [10]. However, there is a general concern that children are sleep deprived and that public health interventions are needed.

Sleep recommendations for children could play an important role in informing public policies, guidelines, interventions, and perhaps most importantly, informing parents and children of healthy sleep behaviors.

Literature referred in the last years to National Sleep Foundation (NSF) recommendations made in 2009 [11]. Recently two groups of experts from NSF [12] and from 
Table 1 Recommended amount of sleep (hours) for age 1 - 14 years according with published recommendations

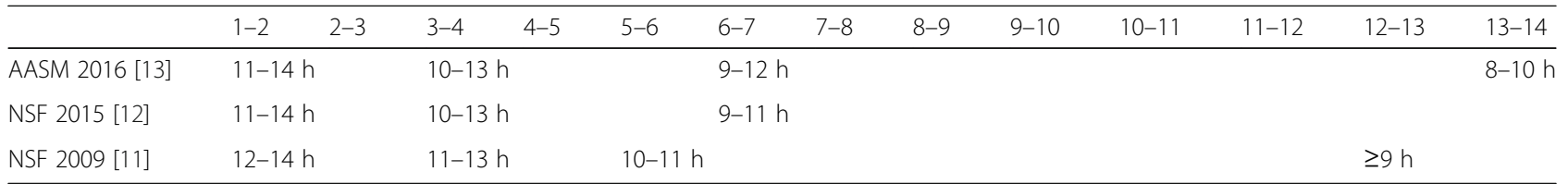

the American Academy of Sleep Medicine (AASM) [13] made separate consensus on sleep recommendations for infants and children that slightly differ from each other. For 4-12 months old children, the AASM suggestion is $12-16 \mathrm{~h}$ per day (including naps) while NSF suggestion is $12-15 \mathrm{~h}$. Table 1 compares AASM recommendations (AASM 2016) with those from NSF (NSF 2009 and NSF 2015), for ages $1-14$ years, showing differences among age groups considered and among proposed limits. In particular, AASM 2016 and NSF 2015 are very similar, while both differs more markedly from NSF 2009, especially for the lower limit proposed for each age group that has been reduced of $1 \mathrm{~h}$.

Sleep recommendations aim to reflect children's "sleep need" or "optimal sleep" duration. Although sleep duration is a necessary component of "optimal sleep," several other factors such as sleep quality, timing, architecture, consistency, and continuity also play an important role. The definition of optimal sleep should consider also the interindividual and intraindividual variability, which can be so great that generalized recommendations may not be of much practical use. Epidemiological studies have acknowledged this individual variability in sleep across development in newborns, infants, and young children [14].

Furthermore, it should be taken into account the significant cross-cultural differences in sleep patterns, sleeping arrangements, and parent-reported sleep problems in children [15]. As a result, there is no single "magic number" for the duration of sleep needed by children of a certain age, and recommendations are always based on a range of hours. Moreover, guidelines on recommended number of hours of sleep are always given in the context of other clues, which parents can use to determine whether their child or adolescent is receiving sufficient sleep, such as not waking spontaneously in the morning, excessive daytime sleepiness, and requiring additional sleep on weekends and during school vacations [16].

Nevertheless, sleep recommendations are issued to help guide optimal sleep on the basis of duration [10].

\section{Impact of recommendations on population}

In this issue of the Journal our group published results of a large survey ("Ci piace sognare" Study) on sleep habits and duration of an Italian population of children

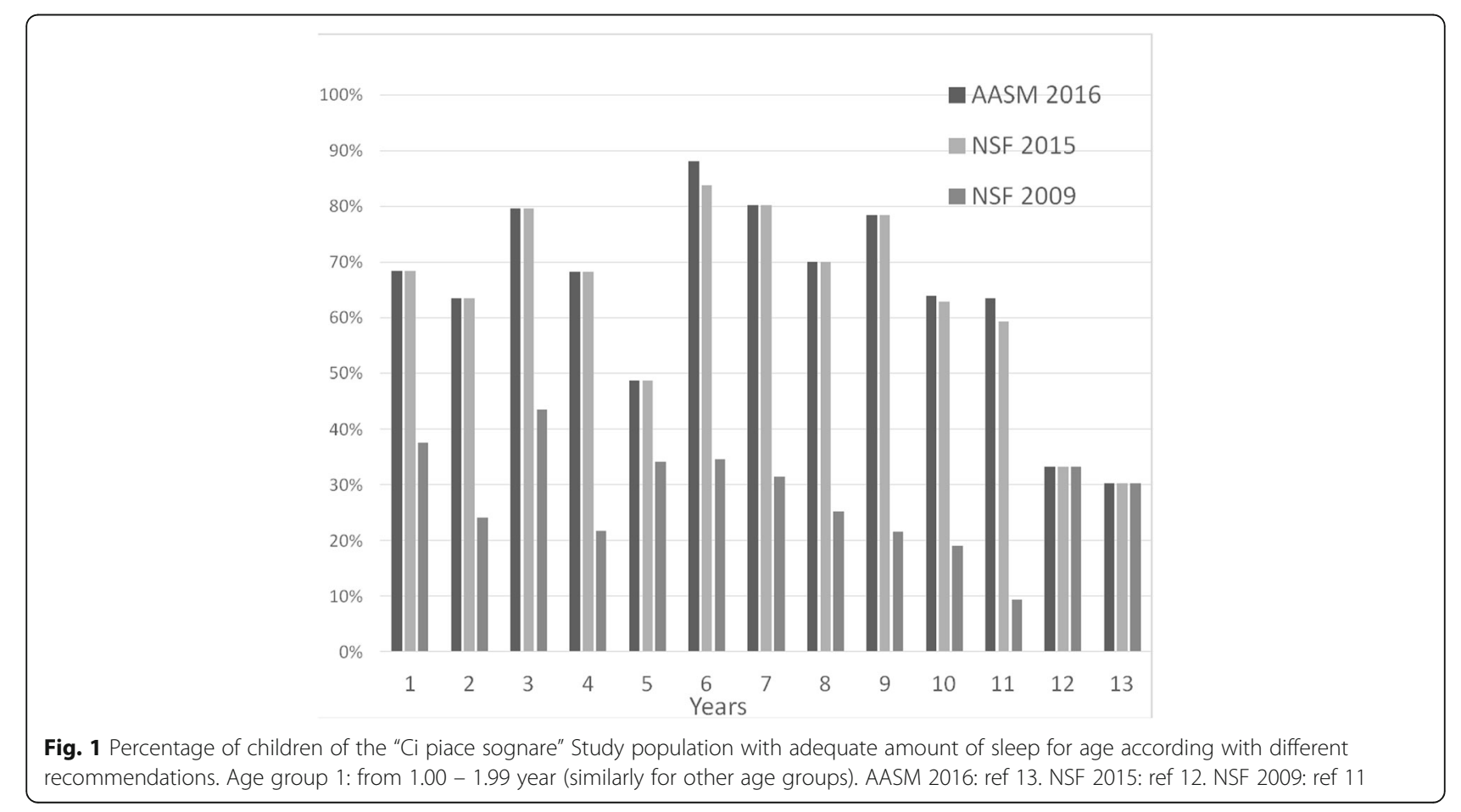


and adolescents aged $1-14$ years old performed in 2015 [17]. We compared the percentages of adequacy of studied population by age obtained by using the cutoff values of NSF 2009, NSF 2015 and AASM 2016 recommendations. Figure 1 reports the results of this comparison. In our sample, we found that about $20-30 \%$ of children do not obtain the adequate amount of sleep that they need in the first 10 years of life. However, the difference is more pronounced in the older age groups and especially in preadolescence where about $70 \%$ of children are not compliant with the recommendations.

These data seem to support the need for the updated references (AASM or NSF 2015) able to better describe a rapidly evolving situation like that of sleep in childhood, emphasizing however the need for a public health policy to inform parents and children on the correct amount of sleep needed [18].

\section{Conclusions}

Although there are still insufficient data to support specific sleep recommendations for children since current sleep recommendations are based largely on expert opinion, the new recommendations seem to reflect clearly the changes in the sleep need of the children and adolescents in the last decades.

However, our recent survey showed that almost $20-30 \%$ of children in the first 10 years of life and about $70 \%$ of preadolescents can be considered in some way "sleep deprived".

Obviously the dramatic changes of the society with the advent of internet and social networking are rapidly changing our habits and our sleep-wake cycle. Therefore, a unified approach by researchers to establish standardized protocols to evaluate optimal sleep across pediatric age groups and especially in preadolescence and adolescence is required, developing sleep restriction/extension protocols, and defining the best age-appropriate measurable tasks for performance.

\section{Abbreviations}

AASM: American Academy of Sleep Medicine; NSF: National Sleep Foundation

\section{Acknowledgements}

Not applicable.

\section{Funding}

None.

\section{Availability of data and materials}

Data sharing not applicable to this article as no datasets were generated or analysed during the current study.

\section{Authors' contributions}

$\mathrm{OB}$ and PB: study design, data analysis, paper writing and editing. All Authors read and approved the final manuscript.

\section{Competing interests}

The authors declare that they have no competing interests.
Consent for publication

Not applicable.

Ethics approval and consent to participate

Not applicable.

\section{Author details}

'Dept of Developmental and Social Psychology, Sapienza University, Roma, Italy. ${ }^{2}$ Family Pediatrician, Azienda Tutela della Salute (ATS), Città

Metropolitana di Milano, Italy. ${ }^{3}$ Via Parada 32, 20854 Vedano al Lambro (MB), Italy.

Received: 28 December 2016 Accepted: 11 January 2017

Published online: 25 January 2017

\section{References}

1. Gruber R, Laviolette R, Deluca P, Monson E, Cornish K, Carrier J. Short sleep duration is associated with poor performance on IQ measures in healthy school-age children. Sleep Med. 2010;11:289-94.

2. Patel S, Hu F. Short sleep duration and weight gain: a systematic review. Obesity. 2008;16:643-53.

3. Hayley AC, Skogen JC, Sivertsen B, Wold B, Berk M, Pasco JA, Øverland S. Symptoms of depression and difficulty initiating sleep from early adolescence to early adulthood: A longitudinal study. Sleep. 2015;38:1599-606.

4. Alfano CA, Zakem AH, Costa NM, Taylor LK, Weems CF. Sleep problems and their relation to cognitive factors, anxiety, and depressive symptoms in children and adolescents. Depress Anxiety. 2009;26:503-12.

5. Gomez RL, Edgin JO. Sleep as a window into early neural development: shifts in sleep-dependent learning effects across early childhood. Child Dev Perspect. 2015;9:183-9.

6. Sadeh A, De Marcas G, Guri Y, Berger A, Tikotzky L, Bar-Haim Y. Infant sleep predicts attention regulation and behavior problems at 3-4 years of age. Dev Neuropsychol. 2015;40:122-37.

7. Matricciani L, Olds T, Petkov J. In search of lost sleep: secular trends in the sleep time of school-aged children and adolescents. Sleep Med Rev. 2012;16:203-11.

8. Gregory AM, O'Connor TG. Sleep problems in childhood: a longitudinal study of developmental change and association with behavioral problems. J Am Acad Child Adolesc Psychiatry. 2002;41:964-71.

9. Friedman NP, Corley RP, Hewitt JK, Wright KP. Individual differences in childhood sleep problems predict later cognitive executive control. Sleep. 2009;32:323-33.

10. Matricciani L, Blunden S, Rigney G, Williams MT, Olds TS. Children's sleep needs: is there sufficient evidence to recommend optimal sleep for children? Sleep. 2013;36:527-34.

11. Mindell JA, Meltzer LJ, Carskadon MA, Chervin RD. Developmental aspects of sleep hygiene: findings from the 2004 national sleep foundation sleep in america poll. Sleep Med. 2009;10:771-9.

12. Hirshkowitz $M$, Whiton $K$, Albert SM, Alessi C, Bruni O, DonCarlos L, Hazen N, Herman J, Katz ES, Kheirandish-Gozal L, Neubauer DN, O'Donnell AE, Ohayon M, Peever J, Rawding R, Sachdeva RC, Setters B, Vitiello MV, CatesbyWare J. National sleep Foundation's sleep time duration recommendations: methodology and results summary. Sleep Health. 2015;1:40-3.

13. Paruthi S, Brooks L, D’Ambrosio C, Hall WA, Kotagal S, Lloyd RM, Malow BA, Maski K, Nichols C, Quan SF, Rosen CL, Troester MM, Wise MS. Recommended amount of sleep for pediatric populations: a consensus statement of the american academy of sleep medicine. J Clin Sleep Med. 2016;12:785-6.

14. Galland BC, Taylor BJ, Elder BE, Herbison P. Normal sleep patterns in infants and children: a systematic review of observational studies. Sleep Med Rev. 2012;16:213-22.

15. Mindell JA, Sadeh A, Wiegand B, How TH, Goh DY. Cross-cultural differences in infant and toddler sleep. Sleep Med. 2010;11:274-80.

16. Owens J. A letter to the editor in defense of sleep recommendations. Pediatrics. 2012;129:987-8.

17. Brambilla P, Giussani M, Pasinato A, Venturelli L, Privitera F, Miraglia del Giudice E, Sollai S, Picca M, Di Mauro G, Bruni O, Chiappini E. Sleep habits and pattern in 1-14 years old children and relationship with video devices use and evening and night child activities. It J Ped. (in press)

18. Blunden S, Galland B. The complexities of defining optimal sleep: empirical and theoretical considerations with a special emphasis on children. Sleep Med Rev. 2014:18:371-8. 